\title{
Radical Scavenging Reaction Kinetics with Multi-Walled Carbon Nanotubes
}

Shuji Tsuruoka ${ }^{\text {a, }}$, Hidetoshi Matsumoto ${ }^{\mathrm{b}}$, Kenichi Koyama ${ }^{\mathrm{a}}$, Eiji Akiba ${ }^{\mathrm{c}}$, Takashi Yanagisawa $^{\mathrm{d}}$, Flemming R. Cassee ${ }^{\mathrm{e}}$, Naoto Saito ${ }^{\mathrm{f}}$, Yuki Usui ${ }^{\mathrm{a}}$, Shinsuke Kobayashi ${ }^{\mathrm{f}}$, Dale W. Porter ${ }^{\mathrm{g}}$, Vincent Castranova ${ }^{\mathrm{h}}$, and Morinobu Endo ${ }^{\mathrm{i}}$

${ }^{a}$ Aquatic Innovation Center, Shinshu University, 4-17-1 Wakasato, Nagano 380-8553 Japan

${ }^{\mathrm{b}}$ Department of Organic and Polymeric Materials, Tokyo Institute of Technology, 2-12-1-S827 Ookayama, Meguro-ku, Tokyo, Japan

${ }^{\mathrm{c}}$ Kuraray Living Co., Ltd., 8-1 Kadota-cho, Osaka 530-8611, Japan

${ }^{\mathrm{d}}$ GSI Creos Corporation, 1-12, Minami-Watada-cho, Kawasaki, Kanagawa, 210-0855, Japan

${ }^{\mathrm{e}}$ National Institute for Public Health \& Environment, Antonie van Leeuwenhoeklaan 9, 3721

Bilthoven, MA, The Netherlands

Institute for Risk Assessment Sciences, Utrecht University, Yalelaan 104, 3584 CM Utrecht, The Netherlands

${ }^{\mathrm{f}}$ Department of Applied Physical Therapy, Shinshu University, School of Health Sciences, 31-1 Asahi, Matsumoto, Nagano, Japan

${ }^{g}$ National Institute for Occupational Safety and Health, Pathology \& Physiology Research Branch, 1095 Willowdale Rd. (M/S2015), 26505-2888 Morgantown, WV, USA

${ }^{\mathrm{h}}$ Department of Basic Pharmaceutical Sciences, West Virginia University, School of Pharmacy, 26506 Morgantown, WV, USA

${ }^{\mathrm{i}}$ Institute of Carbon Science and Technology, Shinshu University, Nagano 380-8553 Japan

\section{Asbtract}


Progress in the development of carbon nanotubes (CNTs) has stimulated great interest among industries providing new applications. Meanwhile, toxicological evaluations on nanomaterials are advancing leading to a predictive exposure limit for CNTs, which implies the possibility to design safer CNTs. To pursue safety by design, redox potential in reactions with CNTs has been contemplated recently. However, the chemical reactivity of CNTs has not been explored kinetically, so that there is no scheme to express a redox reaction with CNTs, though it has been investigated and reported. In addition, reactivity of CNTs is discussed with regard to impurities that consist of transition metals in CNTs, which obfuscates the contribution of CNTs to the reaction. The present work aimed at modeling CNT scavenging in aqueous solution using a kinetic approach and a simple first order reaction scheme. Results show that CNTs follow the redox reaction assumption in a simple chemical system. As a result, the reaction with multi-walled CNTs is semi-quantitatively denoted as redox potential, which suggests that their biological reactions may also be evaluated using a redox potential scheme.

\section{Introduction}

Carbon nanotubes (CNTs) may be useful for various medical, commercial and industrial applications, and designing their structures has recently become an important issue in order to obtain tailor-made performances [1]. At present, their diameter and length are only rudimentarily controllable, while in the laboratory diameter controlled double-walled CNTs (DWCNTs) were synthesized [2,3]. The inner space of CNTs is utilized to deliver particular performances with various particles [4,5]. Industrially, atypical multi-walled CNTs (MWCNTs) are applied and commercialized [6 -11]. Thus, modifications of CNT structures will become an important issue to synthesize and obtain appropriate functionalities and safety in use. Among the challenges with CNTs, particularly MWCNTs, a new and crucial goal will be to design safe CNT structures, while toxicological evaluations on CNTs are advancing leading to a predictive exposure limit for MWCNTs [12]. This groundbreaking challenge 
requires identification of a key mechanism which controls toxicological phenomena [13]. The importance of physicochemical properties is often proposed, but the relative importance of specific properties has not been defined explicitly. Two critical points concerning CNT safety evaluations are summarized as the fiber paradigm and bioactivity, e.g., metal impurities of CNTs [14]. The former not only applies to CNTs but also other nanowires and micro fibers and refers to effects of physical contact with cells and tissues. The latter can be described as chemical reactions on the CNT surface and suggests an intrinsic phenomenon related to biological activities. The metal impurity issue has obscured the contribution of CNTs themselves to bioactivity. Thus, it is necessary to develop a model describing a reaction mechanism for CNTs.

Recent investigations suggest that an intrinsic CNT reaction mechanism may be described by a redox reaction system, because iron is not available on the CNT surface when Fe (III) oxides were formed $[15,16]$. Those impurity effects and their removal are copiously discussed relating to their bioactivities [17-22]. A voltammetric method was used to compare the redox potential of SWCNTs to glassy carbons and associated with the redox potential of CNTs [20]. Nevertheless, Y. Liu, et al. pointed out that those articles were inconclusive and could not be compared to each other [21]. They discussed that CNTs activate the specific molecular signaling associated with oxidative stress activator protein, but also exhibit reactive oxygen species (ROS) scavenging properties. Later, it was reported that because these metals were capsulated into carbon shells, transition metals were not eluted by an acid wash and were not bioavailable [22].

To various degrees, transition metal impurities are usually oxidative to peroxides, while metal oxides are relatively stable. It is known that $\mathrm{Fe}$ (II) or $\mathrm{Fe}^{2+}$ ion generates hydroxyl radicals $(\mathrm{OH} \cdot)$, a form of ROS, in the presence of hydrogen peroxide by the Fenton reaction, and that ROS induce inflammation of tissues. In contrast, $\mathrm{Fe}$ (III) oxide $\left(\mathrm{Fe}_{2} \mathrm{O}_{3}\right)$ and carbide (FeC) do not generate ROS, because Fe (III) cannot be an electron donor except upon 
treatment with a strong reduction agent. Since Fe (II) is supplied not only externally as metal impurities but also internally in a living body and essentially catalyzes peroxide generating hydroxyl radicals, reduction reactions are required to eliminate the radicals. A question is whether the redox potential of CNTs is predictive of ROS generation [13], as CNTs inevitably have chemical reaction sites, for instance, dangling bonds. As of today, it has not been determined if CNT surfaces behave as electron donors or acceptors. If those reaction sites donate electrons to radicals, CNTs become ROS scavengers in an aqueous system.

The present work objectively investigated chemical reactivity and redox potential of MWCNT pseud-quantitatively using its known scavenging ability for hydroxyl radicals. As the chemical reactivity has not been kinetically explored extensively, we hypothesized a simple first order chemical reaction system for MWCNTs, hydrogen peroxide, and hydroxyl radicals, and designed an experimental method to verify the assumption. To embody it, chemical reactions with those components were investigated to eliminate unnecessary disturbances as much as possible. The present studies suggest that the experimental results agree with the assumption, which validates the study of redox potential to evaluate the chemical reactivity of CNTs.

\section{Experimental}

\subsection{MWCNTs}

Two kinds of MWCNTs were used in the present work: cup-stack MWCNTs (CS-MWCNTs) prepared by GSI Creos Corporation (Tokyo, Japan), and Nanocyl NC-7000 MWCNTs obtained from Nanocyl. The average diameter and length of CS-MWCNTs were $80 \mathrm{~nm}$ and 5 $\mu \mathrm{m}$, respectively. Also the average diameter and length of Nanocyl NC-7000 were $9.5 \mathrm{~nm}$ and $1.5 \mu \mathrm{m}$, respectively. The former was provided in order to evaluate the influence to scavenge

performance of chemical components. Since CS-MWCNTs have many graphene edges on 
their surface as shown in Fig. 1, they might be relatively reactive chemically. CS-MWCNTs were characterized in a previous article [26]. The latter was used to measure the intrinsic radical scavenge rate of MWCNTs using a typical MWCNT produced by the catalytic chemical vapor deposition method. To reduce surfactant amount to a minimal concentration against MWCNTs and obtain their good dispersion in water, a specially prepared CNTEC ${ }^{\circledR}$ produced by Kuraray Living Co., Ltd., (Tokyo, Japan) was used as described in Section 2.2.

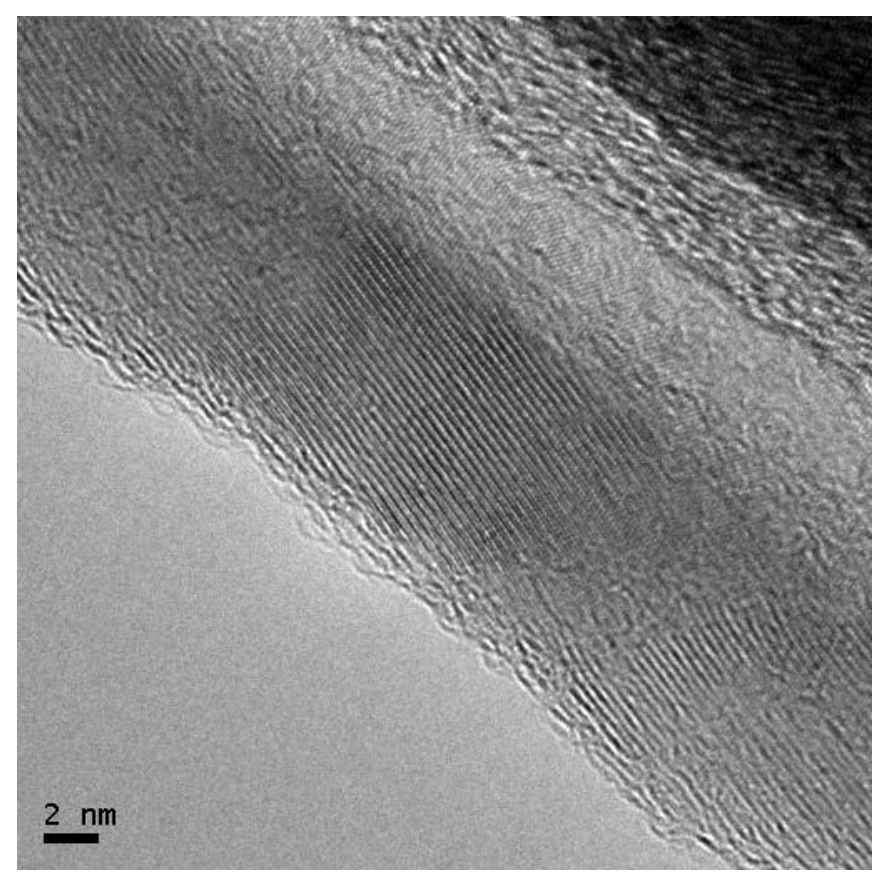

Fig. 1 - An electron microscopy picture of a CS-MWCNT. Graphene layers are stacked and are not parallel to the fiber axis. There are edges of graphene sheets on the surface.

\subsection{Preparation of Mixtures and ESR-DMPO Method}

A measuring mixture consisted of MWCNTs, hydrogen peroxide, ferrous chloride, and 5,5dimethyl-1-pyrriline-1-oxide (DMPO). Hydrogen peroxide (hydrogen peroxide 30.0-35.5 mass\%, Wako Pure Chemical Industries, Ltd. Japan) was diluted to $0.1 \mathrm{M}$ with ultrapure water. The $0.1 \mathrm{M}$ solution was diluted to $1 \mathrm{mM}$ with ultrapure water before use. Ferrous chloride (Iron (II) Chloride Tetrahydrate, Wako Pure Chemical Industries, Ltd. Japan) was dissolved in ultrapure water to $15.7 \mathrm{mM}$. This solution was also diluted 100 times before use. 
Frozen DMPO (Dojindo Laboratories, Kumamoto Japan) was thawed at room temperature and diluted to $100 \mathrm{mM}$ with ultrapure water. The DMPO solution was prepared each time and disposed within 24 hours after preparation. Surfactant for CS-MWCNTs was sodium dodecyl benzensulfonate (SDS) (Kanto Chemical Co., Inc. Japan) and was diluted to $45.9 \mathrm{mM}$ with ultrapure water.

CNTEC $^{\circledR}$ was made of polyester fibers coated with 12 wt\% Nanocyl NC-7000 in dry condition. Weight ratio of concentration of the surfactant to MWCNTs of CNTEC ${ }^{\circledR}$, that was specially prepared for, was fixed at 26.2 to 100 in dry condition. $0.1 \mathrm{~g}$ CNTEC fibers were dispersed into $50 \mathrm{~g}$ of ultrapure water, which was sonicated for 30 minutes in a ultrasonic bath. The mixture was filtered with a Whatman filter paper (Whatman 42 with pore size at 2.5 $\mu \mathrm{m})$ to remove polyester fibers and large agglomerates of MWCNTs. This solution was named as Solution A that included $0.13 \mathrm{wt} \%$ of MWCNTs after drying the solution. Solution A was filtered with a Whatman filter paper $(\mathrm{GF} / \mathrm{F}$ with pore size $0.7 \mu \mathrm{m})$ and then a Milipore filter (MF-Milipore GSWP 09000m with pore size at $0.22 \mu \mathrm{m}$ ). This solution, named Solution B, included $0.036 \mathrm{wt} \%$ of MWCNTs. The procedure gave an advantage to balancing the surfactant interference despite MWCNT concentration alterations. These solutions were used instead of CS-MWCNTs that were dispersed into the surfactant solution.

In all measurement, peroxide concentration was excessed.

\subsection{Electron Spin Resonance Measurement}

All solutions were mixed and measured at room temperature with Electron Spin Resonance (ESR) (JES-FA100, JEOL). ESR settings were: frequency $9415.404 \mathrm{MHz}$, power $0.998 \mathrm{~mW}$, field center $335 \mathrm{mT}$, sweep time $2 \mathrm{~min}$., width +/- $5 \mathrm{mT}$, and modulation frequency $100 \mathrm{kHz}$. All measurements were conducted within 5 minutes after mixing all of those solutions. The details were reported in a previous article [26]. 
ESR spectra were normalized using Mixture B in Table 1 with $0.1 \mathrm{ml} \mathrm{CNT}$ solution for all of the CS-MWCNT measurements. With Nanocyl NC-7000, Mixture A in Table 1 without surfactant was used. ESR measurement results were obtained as relative values to a reference. In the present work, radical concentration in a reference solution or specified MWCNT mixture was described using the normalized form;

Scavenging ratio $=[$ ESR signal of a sample $/$ ESR signal of a reference $]$

$$
=[\text { Radical concetration of a sample/Radical concentration of a reference }]
$$

Since the ratio is one at $\mathrm{MWCNT}=0$, radical concentration with a change of $\mathrm{CNT}$ concentration was expressed as radical concentration ratio to the reference. On the other hand, radical scavenging rate is described as;

Scavenging rate $=\{1-($ Scavenging ratio $)\}$

ESR spectra were normalized using Mixture B in Table 1 with $0.1 \mathrm{ml} \mathrm{CNT}$ solution for all of the CS-MWCNT measurements. With Nanocyl NC-7000, Mixture A in Table 1 without surfactant was used. Thus, the scavenge ratio and rate represent the normalized hydroxyl radical concentration relative to the reference and the normalized hydroxyl radical concentration amount scavenged in a solution, respectively. All of the samples were measured at least five times and arithmetically averaged except the lowest and highest values. In the present work, a buffer to control solution $\mathrm{pH}$ was not added because the buffer apparently affects the reaction and reactive components were in the aqueous solution. $\mathrm{pH}$ measurement was not conducted during ESR-DMPO measurement because it cannot be physically measured during the ESR spectrum measurements.

\section{Results and Discussion}

\subsection{Reaction Kinetics Hypothesized}

According to recent findings, MWCNTs scavenge ROS [23-26]. All of those reports hinted that the reaction occurs at dangling bonds on CNT surfaces and MWCNTs supposedly act as 
electron donors or, at least charge is transferred from those dangling bonds to radicals. Petersen et al. reported that SWCNTs also scavenge hydroxyl radicals by electron transfer [27]. Peng et al. found that MWCNTs attached with cadmium sulfide (CdS) were electron acceptors and catalyzed conversion of water to hydrogen (and inevitably oxygen) in a photoreaction as a simulated photosynthetic reaction, where radical formation and degeneration were implicitly included [28]. This indicates that MWCNTs can be both electron acceptors and donators in redox reactions depending on their relative chemical potentials. If redox potential is hypothesized for MWCNTs, they may decrease oxidant-induced inflammation of tissues, though the actual condition surrounding MWCNTs is complicated. One would be able to stoichiometrically predict oxidant stress once the redox potential of MWCNTs is determined in a reaction system. To conduct and specify CNT behavior in aqueous solution, it is necessary to model it using a simple first order reaction profile for CNTs as the first step.

We hypothesize the following chemical reaction equations with MWCNTs and hydrogen peroxide (Fig. 2). First, in the light of the fact that a description of the Fenton reaction has not been agreed upon completely, a simple system consisting of hydrogen peroxide and Fe (II) can be written to characterize the present experimental system specifically as follows [29, 30].

$$
\begin{aligned}
& \mathrm{Fe}^{2+}+\mathrm{H}_{2} \mathrm{O}_{2} \rightarrow \mathrm{Fe}^{3+}+\mathrm{HO}^{-}+\mathrm{HO} \\
& \mathrm{Fe}^{3+}+\mathrm{H}_{2} \mathrm{O}_{2} \rightarrow \mathrm{Fe}^{2+}+\mathrm{H}^{+}+\mathrm{HOO} \\
& \mathrm{Fe}^{3+}+\mathrm{HOO} \cdot \rightarrow \mathrm{Fe}^{2+}+\mathrm{H}^{+}+\mathrm{O}_{2}
\end{aligned}
$$

These equations can be summarized as:

$$
3 \mathrm{H}_{2} \mathrm{O}_{2} \rightarrow 2 \mathrm{H}_{2} \mathrm{O}+\mathrm{O}_{2}+2 \mathrm{HO}
$$


According to previous reports [12, 20, 22-25], it is agreed that CNTs scavenge hydroxyl radicals in an aqueous solution with hydrogen peroxide experimentally. Assumed reaction sites on CNT surface including dangling bonds are denoted as $D n$ that acts as if they were single molecules. As long as Eq. (4) is true, a necessary condition to satisfy it with radical scavenging must become an equation as:

$2 \mathrm{Dn}+2 \mathrm{HO} \cdot \rightarrow 2 \mathrm{Dn}^{+}+\mathrm{H}_{2}+\mathrm{O}_{2}$

Accordingly, Eqs. (4) plus (5) give the following equation:

$3 \mathrm{H}_{2} \mathrm{O}_{2}+2 \mathrm{Dn} \rightarrow 2 \mathrm{Dn}^{+}+2 \mathrm{H}_{2} \mathrm{O}+\mathrm{H}_{2}+2 \mathrm{O}_{2}$

Eq. (5) indicates that reaction sites donate electrons to hydroxyl radicals finitely. This agrees with the assumption of $\mathrm{H}+$ or $\mathrm{OH}$ radical generation by electron acceptance on $\mathrm{CNT}$ s by Peng et al. [28]. In Eq. (6), the reaction rate constant should become "1" if the concentration of Dn is large enough and dominates compared to that of $\mathrm{H}_{2} \mathrm{O}_{2}$ from Eqs. (S5) and (S6) in Supplemental. This suggests that this experimental condition must be avoided. Furthermore, the equations predict that $\mathrm{CNT}$ amount, or mole equivalent of the number of reaction sites, is necessarily smaller than that of hydrogen peroxide. Thus, while mole equivalent of CNTs or reaction sites have not been determined, a concentration ratio of hydrogen peroxide to CNTs should be sought in an experiment.

Eq. (5) requires ones to measure concentrations of hydrogen and oxygen in a scavenging reaction in order to verify the Fenton reactions. However, it is experimentaly impossible to measure those concentrations in situ because of measuring system of the ESR equipment and its measuring cell structure. Fortunately Eq. (5) is a fictitious reaction to deduce Eq. (6) so that Eq. (5) is regarded as an intermediate reaction. Although the Fenton reaction gives many routes of reaction steps, it can be simplified in such manner. 


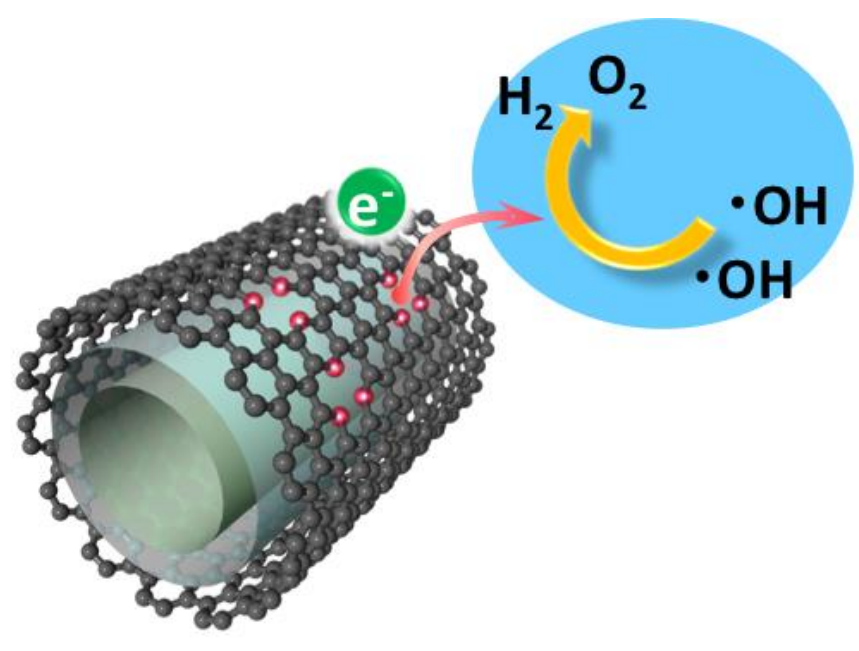

Fig. 2 - A schematic diagram to illustrate hypothesized reaction kinetics of hydroxyl radicals at a reaction site of MWCNTs. To make it easy to see the assumption concept, it is illustrated as if the reaction takes place at a dangling bond. Reaction sites donate electrons to hydroxyl radicals and result in hydrogen and oxygen as denoted in Eq. (6).

\subsection{Influences of Chemicals in a Reaction System}

Before conducting chemical tests to investigate whether Eq. (6) is appropriate to describe the present chemical reaction, it is necessary to investigate influences by chemicals in an ROS measurement. This has not been pursued previously, because the present approach with chemical kinetics had not been proposed nor systematically explored. In addition, it was reported that chemicals in similar systems significantly affect ESR-DMPO measurement [31]. We conducted a series of tests using CS-MWCNTs (Fig. 1), because they have many edges of graphene that are relatively reactive in comparison with highly crystallized CNTs [26]. Fig. 3a shows that the radical scavenging rate varies with a concentration change of surfactant without CS-MWCNTs, where Mixture A in Table 1 was used. A reference solution was at 0 $\mathrm{mM}$ of surfactant of Mixture A in Table 1. Results show that hydroxyl radicals are scavenged proportional to a surfactant concentration. Fig. 3a apparently means that surfactant scavenges radicals. Fig. $3 b$ shows the radical scavenge rate with a concentration change of MWCNTs in a fixed concentration of surfactant at $0.918 \mathrm{mM}$ in a solution, where Mixture B was used in 
Table 1. Likewise the reference solution was at $0 \mathrm{wt} \%$ of MWCNTs of Mixture B in Table 1. As a scavenge rate of hydroxyl radicals proportionally corresponds to a surfactant concentration according to Fig. 3a, Fig. $3 b$ indicates that a scavenge rate is proportional to a concentration change of CS-MWCNTs at a fixed surfactant concentration, where the surfactant contribution is relatively low. This suggests that the ESR-DMPO method can measure radical concentration changes corresponding to a CS-MWCNT concentration change. However, an intrinsic CS-MWCNTs scavenge performance cannot be measured using this method because the respective contributions of CS-MWCNTs and surfactant are not distinguished individually. Fig. $3 \mathrm{c}$ demonstrates the scavenge ratio with a change of hydrogen peroxide concentration at a fixed concentration of surfactant without CS-MWCNTs. The scavenge ratio does not change with a hydrogen peroxide concentration change, which indicates that hydroxyl radical generation depends on duration time after mixing those chemicals rather than hydrogen peroxide concentration under the proposed experimental condition. It agrees with previous literature [29, 30]. Therefore, surfactant is specifically a major influence factor in the present chemical reaction system. It is necessary to minimize the surfactant concentration to determine intrinsic MWCNT radical scavenging performance.

In our radical scavenging tests with MWCNTs, $\mathrm{pH}$ measurements were omitted. On one hand, there is physical obstruction in which DMPO adduct has a very short lifetime and the measuring cell cannot be equipped with a $\mathrm{pH}$ cell inside due to physical constraints. It did not allow one to measure $\mathrm{pH}$ in situ. On the other hand, interaction among the buffer chemicals, MWCNTs, and DMPO is complicated and cannot be predicted. Reaction sites on MWCNTs might react with phosphate and DMPO could attach on the CNT surface [32]. It is noted that $\mathrm{pH}$ of the solution mixture just before ESR measurement was approximately $\mathrm{pH}$ 6.5; that was almost equal to that of ultrapure water used. It is regarded that water stabilized $\mathrm{pH}$ due to the very low concentration. It would be optimal to estimate or measure the solution $\mathrm{pH}$ during ESR-DMPO spectra measurement. 
$\mathbf{a}$

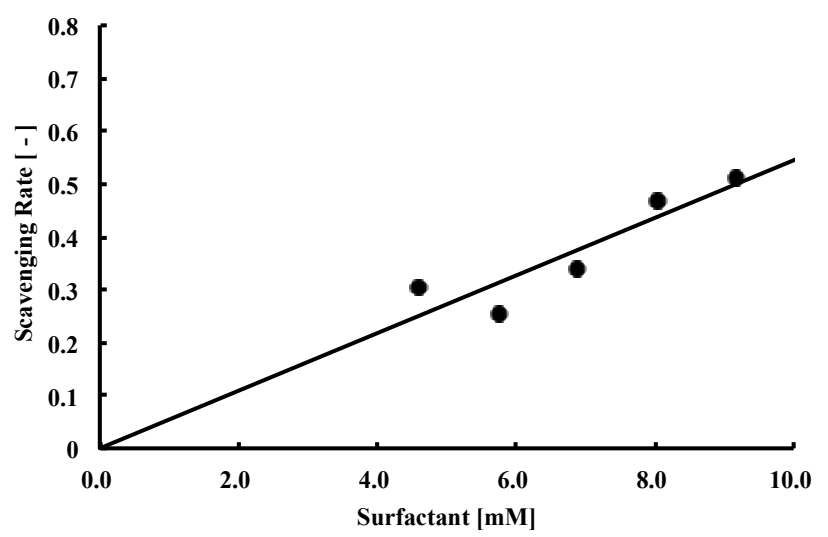

b

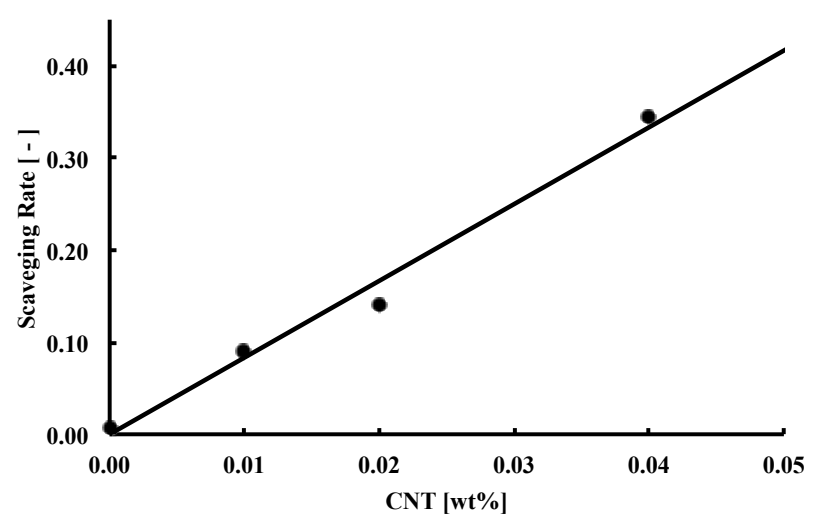

c

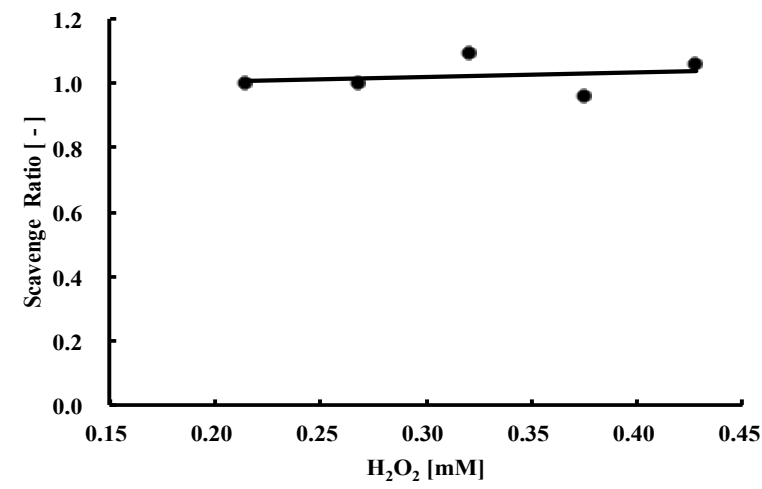

Fig. 3 - Influences of chemical components in the scavenge reaction system. Vertical axis shows the scavenge rate of hydroxyl radicals that were generated by the Fenton reaction with hydrogen peroxide. (A) Influence of surfactant without CS-MWCNTs. The scavenge rate is proportional to surfactant concentration. (B) A scavenge rate change with a change of CSMWCNT concentration at a fixed surfactant concentration of $0.918 \mathrm{mM}$. Scavenge rate proportionally corresponds to the MWCNT concentration change. (C) The scavenge ratio with a change of hydrogen peroxide concentration in fixed concentrations of $\mathrm{FeCl}_{2}$ and 
surfactant in Mixture B without CS-MWCNTs in Table 1. It is apparent that radical concentration is constant at the measuring time in the solution.

\subsection{Scavenging Performance Measurements with the Minimal Amount of Surfactant and Intrinsic Redox Potential of MWCNTs}

As mentioned above, because of the influence of surfactant scavenging, performance measurements were conducted using the minimal amount of surfactant with MWCNTs to determine the intrinsic contribution of MWCNTs to radical scavenging. Fig. 4 shows a change in scavenge rate with a change of MWCNT solution volume, where Solutions A or B was added into water. This figure clearly shows that the radical scavenging depends on MWCNT concentration. In this procedure, surfactant concentration in solutions A and B was identical. Because those solutions were diluted further with ultrapure water and hydrogen peroxide in the measurement, surfactant concentration was two to three digits lower than that of Mixture A or B that were prepared using a conventional method with surfactant. Since surfactant amount was proportional to the MWCNT concentration that was low, the influence of surfactant was believed negligible in Solutions A and B according to Fig. 3a. Fig. 4 demonstrates that the maximum of hydroxyl radicals scavenging rate depends on MWCNT concentration and have their plateau points. This result suggests that the number of reaction sites was significantly different between those solutions. 


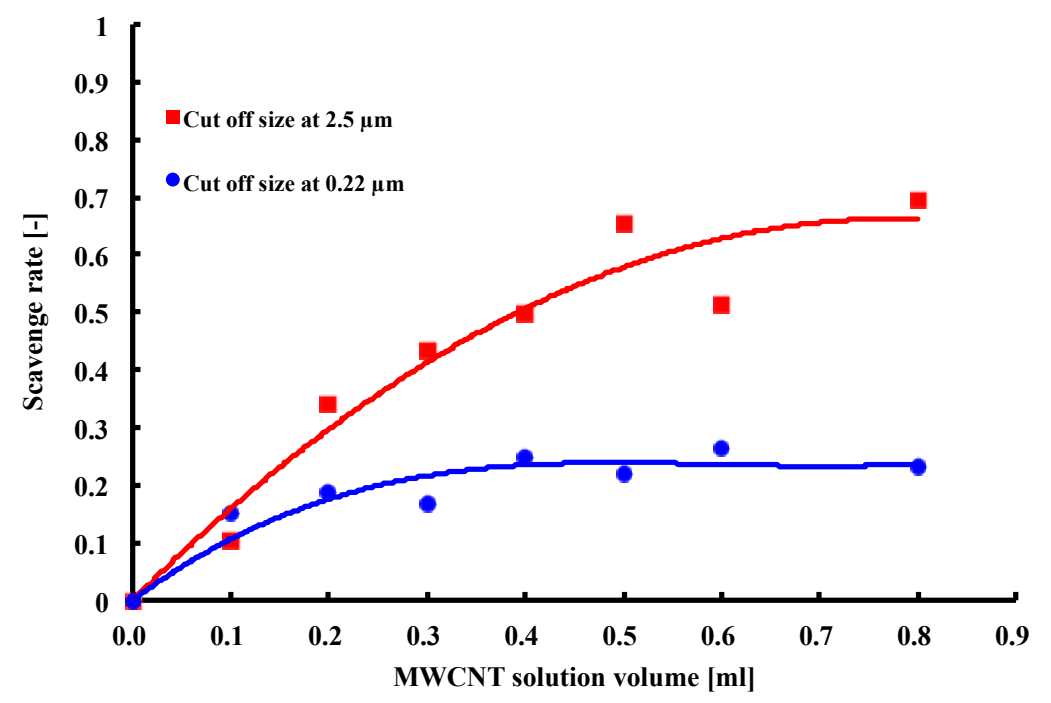

Fig. 4 - Radical scavenge rate with a volume change of MWCNT solutions A and B. Solutions A and B are filtered at 2.5 and $0.22 \mu \mathrm{m}$ to control MWCNT weight concentration, respectively. Those fitting curves are binominal for Solution A and section three approximations for Solution B, respectively. From those fitting curves, equilibrium points of Solutions A and B are at 0.78 and 0.38 , respectively.

Table 1. Solution mixture components for CS-MWCNTs

Amount of solutions taken $[\mathrm{ml}]$

\begin{tabular}{cccccccc}
\hline Solutions & $\mathrm{FeCl}_{2}$ & $\begin{array}{c}\text { CNTs in } \\
\text { surfactant }\end{array}$ & DMPO & Surfactant & $\mathrm{H}_{2} \mathrm{O}_{2}$ & $\begin{array}{c}\text { Ultrapure } \\
\text { water }\end{array}$ & $\begin{array}{c}\text { Total } \\
\text { volume }\end{array}$ \\
\hline $\begin{array}{c}\text { Mixture } \\
\text { A }\end{array}$ & 0.4 & None & 0.4 & $0.4-0.8$ & 0.4 & Balance & 2.0 \\
$\begin{array}{c}\text { Mixture } \\
\text { B }\end{array}$ & 0.4 & $0-0.4$ & 0.4 & Balance & 0.4 & 0.4 & 2.0 \\
\hline
\end{tabular}

In comparison with the experimental results discussed in the previous section, the curvature in Fig. 4 is apparently different from that in Fig.3b. Although it was true that size distributions of MWCNTs in Solutions A and B were not identical after passing through those filters, the tendency of those curves was alike. Results are consistent with a previous report in which the size difference of particular MWCNTs did not significantly effect the scavenging 
characteristics though surface morphological difference did [26]. Another report used MWCNT weight concentration in the horizontal axis instead of volumetric concentration used in Fig. 4. A single smooth line resulted when those results were plotted against each other [31]. From those facts, it is suggested that the scavenging reaction is proportional to surface area of MWCNTs or the number of reaction sites. Besides, Fig. 4 indicates that scavenge rate does not increase as like as straight line in Fig. 3a, or the first order reaction to CNT concentration. Even though peroxide was excessive in quantity, radicals were generated but not so fast. Therefore, the scavenge rate exhibits a plateau. It means there is an equilibrium point by an unknown mechanism. However this is not the target of the present study. Thus, Eq. (5) is an intermediate reaction simply given to derive Eq. (6) with the Fenton reactions.

In Fig. 5 all plots measured with Solutions A and B are summarized together. The solid line is calculated by Eq. (7) as;

$\mathrm{S}_{\text {rad }}=-q \ln \left|C_{D n}+s\right|+q\left(C_{D n}+s\right)+r$

,where $S_{\text {rad }}$ and $C_{D n}$, are scavenging ratio and MWCNT concentration in a mixture. The detail of definitions is given in the Supplemental. Note that Eq. (7) is equivalent to Eq. (S8') in Supplemental. In Eq. (7) $q, r$, and $s$ are arbitrary constants and were calculated using a “Solver" function of Microsoft Excel (Microsoft ${ }^{\circledR}$ Excel $^{\circledR}$ for Mac2011, Version 14.3.9) as 0.14936, 0.00000, and 0.00105, respectively. Fig. 5 clearly shows that the scavenging reaction ratio, or hydroxyl radical concentration ratio measured agrees with the solid line practically, which indicates that the hypothesis in Eq. (6) is appropriate to denote the reaction system. 


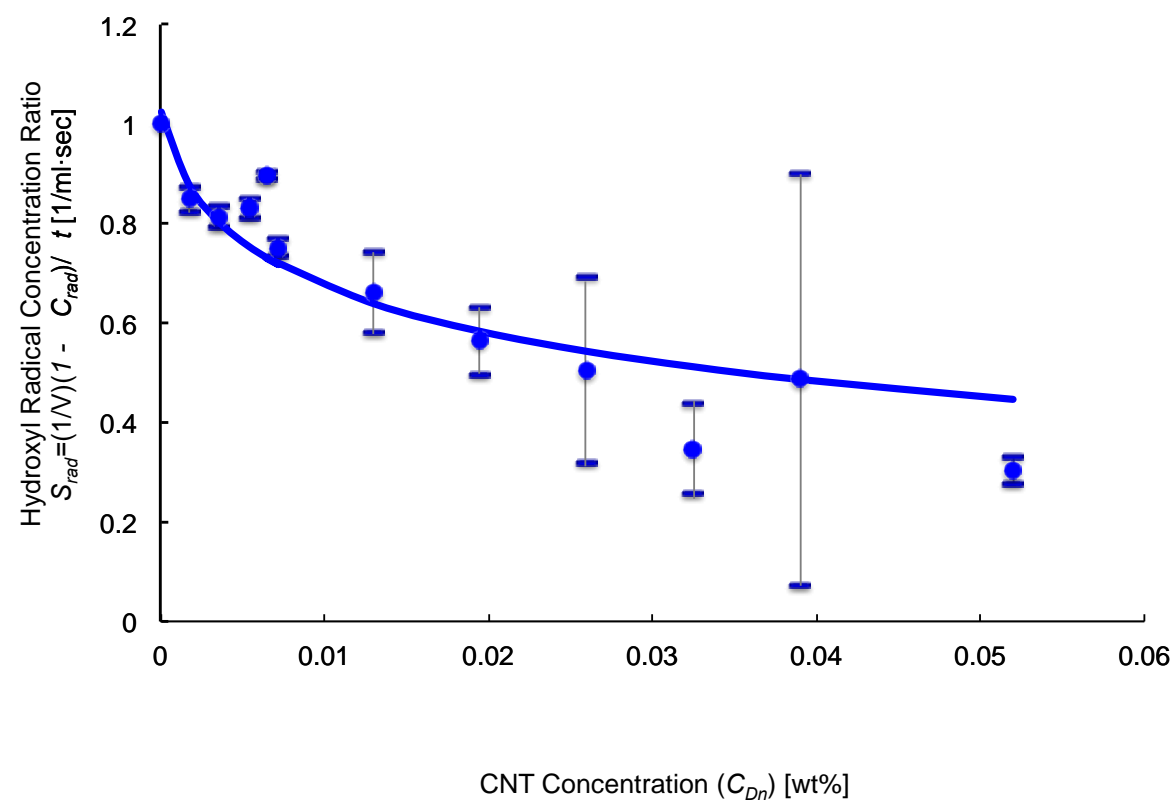

Fig. 5 - A change of hydroxyl radical concentration $S_{\text {rad }}$ in the solution with a change of MWCNTs weight concentrations $C_{D n}$. The solid line is calculated using Equation (7).

Standard deviations of those plots by measurement are indicated with vertical bars.

Fig. 5 evidently shows that the experimental result agrees with Eq. (7). In Fig. 4, on one hand, the results are individually plotted based on those prepared MWCNT solutions in order to show CNT concentration dependency. In Fig. 5, on the other hand, all plots are processed together with a change of CNT weight concentration. The former sets forth the radical scavenging reaction depending on MWCNT surface amount. It is regarded as a technique to detect the reaction rates at very low concentrations of MWCNTs without a change of the other ingredients in the solution. The latter is used to analyze the reaction kinetics. Those measurement standard deviations tend to be small at the lower MWCNT concentrations. It is probably that radical scavenging by surfactant may become significant at the higher MWCNT concentrations as the surfactant concentration is proportional to MWCNT concentration. It is necessary to look for a method to determine reaction rates of hydroxyl radicals-DMPO and hydroxyl radicals-surfactant to verify the point. 
The plateau point is supposed as a pseudo-equilibrium point in this particular reaction system, and may be related to the number of reaction sites of MWCNTs. However, when Eq.

(7) is expanded using Taylor expansion, it is rewritten, if $C_{D n}$ is large enough, as;

$$
\begin{aligned}
S_{\text {rad }} & =-q\left\{2\left(C_{D n}+\frac{C_{D n}{ }^{3}}{3}+\cdots+\frac{C_{D n}{ }^{2 n+1}}{2 n+1}+\cdots\right)\right\}+q C_{D n}+r \\
& =-q\left(C_{D n}\right)+\cdots+\frac{C_{D n}{ }^{2 n+1}}{2 n+1}+\cdots
\end{aligned}
$$

Thus, $S_{r a d}$ is to be the infinite number and does not have an equilibrium point at the large $C_{D n}$, while it has an inflection point. It is considered that Eq. (7) may hold true for a condition at low MWCNT or reaction site concentration having a pseud-plateau point. It means that the scavenging ratio becomes large at a high MWCNT concentration. At present, as mentioned above, this cannot be practically verified since the higher MWCNT concentration brings a greater influence of surfactant, and consequently surfactant conceals the intrinsic scavenging activity by MWCNTs. It is required to develop a technique to disperse a large amount of MWCNTs at a very low concentration of surfactant. However, the intrinsic behavior of MWCNTs can be sought at very low concentrations of components. Differentiating Eq. (7) and setting to zero, it gives a pseudo-equilibrium point at which a slope of Eq. (7) is horizontal. To solve the equation, $C_{D n}=s=0.9985$, and $S_{r a d}=q=0.14936$. This result gives an answer to Eq. (S2); however, it does not specify pseudo-equilibrium constants of $K_{1}$ or $K_{2}$, because actual peroxide concentration in the chemical reaction system is not dynamically determined in the present procedure. It is necessary to seek and develop a measuring method for hydrogen peroxide in the solution in-situ and obtain those constants. Even in a view of them, Eq. (7) should be applied to radical scavenging ability of MWCNTs and their bioavailability evaluations. Here, it has to be determined whether given nano carbons are to be electron acceptors or donators. Krusic et al. specified that fullerenes were endohedral and electron acceptors [33]. On the other hand, physical properties of CNTs are significantly 
different from that of fullerenes, and particularly energy bands and DOS of CNTs are unique because of the cylindrical structure and chirality [34]. In addition, Ullah et al. proposed a concept of "Charge carrier transport mechanism" and discussed that electron transfer of semiconductive materials is relative [35]. Furthermore, Shi et al. pointed out that CNTs can either donate or accept electrons based on an electron transfer mechanism [36]. Since results of the present work correspond to the previous reports [23-27] and are not inconsistent with those discussions, the assumption of electron donation is reasonable. Thus, Eq. (7) is deemed to be appreciable to those evaluations. Figs. 4 and 5 show that reaction kinetics between MWCNTs and hydroxyl radicals agree with Eq. (6), that is, MWCNTs donate electrons to those radicals.

Eq. (7) implies that a high concentration of MWCNTs infinitely scavenge hydroxyl radicals, but scavenging ability by MWCNTs is obviously not proportional to their concentration. Considering those points with CNT surface structure, the results support our hypothesis semiquantitatively though a pseudo-equilibrium constant is not specified uniquely. One should consider calculating the constant if a CNT chirality gives a particular electron energy distribution and density of state specifically in case of thinner diameter [37]. As electron energy distribution and DOS for thicker diameter MWCNTs indicate no significant differences [38], a relationship between redox potential of MWCNTs and chirality has to be clarified with thinner MWCNTs. Furthermore, it is required to investigate if Eq. (6) is reversible or kinetically represent an equilibrium condition. Eq. (6), for example in a biological reaction, predicts that induction of tissue inflammation after exposure to MWCNTs increases in a long period test in which electrons in MWCNTs are depleted, unless the living body can supply electrons to MWCNTs. It has been shown that pulmonary inflammation rapidly increases in the week after exposure to MWCNTs and gradually decreases to a normal condition within a month [39]. The present report suggests that the inflammation decrease may be related to the redox potential. Of interest is whether long period exposures show a 
rebound of inflammation. About electron supply to CNTs, Petersen et al. implies that particular biological reactions give electrons to CNTs [27]. To investigate the biological reaction kinetically, it is necessary to elucidate the Fenton reaction and reactions of biological molecules in the living body. Thus, MWCNTs may have redox potential, while their reactivity as electron acceptors must be proved using an alternative way [40]. Once redox potential of MWCNTs is determined, MWCNT intrinsic toxicity via reactive oxygen species can be estimated in tissues chemically using their physicochemical properties and surrounding conditions. Thus, it may be possible that redox potential of MWCNTs could predict biological responses if reaction conditions around MWCNTs were determined.

\section{Conclusion}

A chemical kinetics scheme to explain the hydroxyl radical scavenging mechanism with MWCNTs is proposed and proven by experiments in a simple chemical system with MWCNTs. Theoretical calculations agree with the experimental results. Surfactant was specified as an interfering factor in the present reaction system. Minimizing surfactant concentration allowed demonstration of the intrinsic behavior of MWCNTs in the system. MWCNTs behave as electron donors through their reaction sites, which is a reason why MWCNTs are ROS scavengers. While it is predicted that surface morphology of MWCNTs can be characterized using chemical reactions on the surface, the present work clearly shows that experimental results agree with chemical kinetics assumed and previous reports. It suggests that this new approach may allow one to estimate toxic reactions based on chemical kinetics using phyisicochemical properties of MWCNTs._Although it is necessary to determine the mole equivalent number of MWCNTs to calculate the absolute reaction and equilibrium constants, a model using redox potential and chemical kinetics may predict the intrinsic chemical reactivity of the MWCNT surface and, therefore, be applied to design safer CNT structures. 


\section{Acknowledgements}

This work is a part of the research program "Development of innovative methodology for safety assessment of industrial nanomaterials" supported by the Ministry of Economy, Trade and Industry (METI) of Japan. ST, KK, and UY were supported by the Exotic Nanocarbon Project, Japan Regional Innovation Strategy Program by the Excellence, JST (Japan Science and Technology Agency). We would like to thank Dr. K. Fujisawa for preparation of graphic figure for Figure 1.

\section{Reference}

[1] P. Eklund, P. Ajayan, R. Blackmon, A.J. Hart, J. Kong, B. Prashan, A. Rao, A. Rinzler, WTEC Panel Report on International assessment of research and development of carbon nanotubes manufacturing and applications. World Technology Evaluation Center, Inc. 2007.

[2] M. Endo, H. Muramatsu, T. Hayashi, Y.A. Kim, M. Terrones, M.S. Dresselhaus, Nature 2005, 433, 476.

[3] H. Muramatsu, T. Hayashi, Y.A. Kim, D. Shimamoto, M. Endo, V. Meunier, B.G. Sumpter, M. Terrones, M.S. Dresselhaus, Small 2009, 5, 2678-2682.

[4] H. Muramatsu, D. Shimamoto, T. Hayashi, Y.A. Kim, M. Endo, M. Terrones, M.S. Dresselhaus, Advanced Materials 2011, 23, 1761-1764.

[5] T. Fujimori, A. Morelos-Gómez, Z. Zhu, H. Muramatsu, R. Futamura, K. Urita, M. Terrones, T. Hayashi, M. Endo, S.Y. Hong, Y.C. Choi, D. Tománek, K. Kaneko, Nat. Commun. 2013, 4, Article number: 2162.

[6] M. Endo, Y.A. Kim, T. Hayashi, T. Fukai, F. Oshida, M. Terrones, T. Yanagisawa, S. Higaki M.S. Dresselhaus, Appl. Phys. Lett. 2002, 80, 1267-1269.

[7] T. Hayashi, T.C. O'Connor, K. Higashiyama, K. Nishi, K. Fujisawa, H. Muramatsu, Y.A. 
Kim, B.G. Sumpter, V. Meunier, M. Terronese, M. Endo, M. Nanoscale 2013, 5, 10043 10670.

[8] M. Endo, T. Noguchi, M. Ito, K. Takeuchi, T. Hayashi, Y.A. Kim, T. Wanibuchi, H. Jinnai, M. Terrones, M.S. Dresselhaus, Adv. Funct. Mater. 2008, 18, 3403-3409.

[9] M. Endo, K. Takeuchi, T. Noguchi, Y. Asano, K. Fujisawa, Y.A. Kim, T. Hayashi, H. Ueki, S. Iinou, Ind. Eng. Chem. Res. 2010, 49, 9798-9802.

[10]C. Sotowa, G. Origi, M. Takeuchi, Y. Nishimura, K. Takeuchi, I.Y. Jang, Y.J. Kim, T. Hayashi, Y.A. Kim, M. Endo, M.S. Dresselhaus, ChemSusChem. 2008, 1, 911-915.

[11]S. Tsuruoka, B. Fugetsu, F. Khoerunnisa, D. Minami, K. Takeuchi, M. Fujishige, T. Hayashi, Y.A. Kim, K.C. Park, M. Asai, K. Kaneko, M. Endo, Mater. Express 2013, 4, 21-29.

[12] J. Howard, Current Intelligence Bulletin 65: Occupational Exposure to Carbon Nanotubes and Nanofibers, DHHS (NIOSH) Publication No. 2013-145, 2013.

[13] S. Tsuruoka, F.R. Cassee, V. Castranova, Part. Fibre Toxicol. 2013, 10:44.

[14] K. Donaldson, C.A. Poland, Curr. Opin. in Biotechnol. 2013, 24, 724-734.

[15]L. Guo, D.G. Morris, X. Liu, C. Vaslet, R.H. Hurt, A.B. Kane, Chem. Mater. 2007, 19, $3472-3478$.

[16]C. Ge, F. Lao, W. Li, Y. Li, C. Chen, Y. Qiu, X. Mao, B. Li, Z. Chai, Y. Zhao, Anal Chem. 2008, 80, 9426-9434.

[17]M. Pumera, Langmuir 2007, 23, 6453-6458.

[18] M. Pumera, Y. Miyahara, Nanoscale 2009, 1, 260-265.

[19]C. Ge, Y. Li, J-J Yin, Y. Liu, L. Wang, Y. Zhao, C. Chen, NPG Asia Mater. 2012, 4, 1-10.

[20]R.J. Toh, A. Ambrosi, M. Pumera, Chem. - Eur. J. 2012, 18, 11593-11596.

[21] Y. Liu, Y. Zhao, B. Sun, C. Chen, Acc. Chem. Res. 2013, 46, 702-713.

[22] X. Liu, L. Guo, D. Morris, A.B. Kane, H. Hurt, Carbon 2008, 46, 489-500. 
[23]I. Fenoglio, M. Tomatis, D. Lison, J. Muller, A. Fonseca, J.B. Nagy, B. Fubini, Free. Radical Biol. Med. 2006, 40, 227-1233.

[24]I. Fenoglio, G. Greco, M. Tomatis, J. Muller, E. Raymundo-Piñero, F. Béguin, F.; Fonseca, J.B. Nagy, D. Lison, B. Fubini, Chem. Res. Toxicol. 2008, 21, 1690-1697.

[25] A.R. Murray, E. Kisin, S.S. Leonard, S.H. Young, C. Kommineni, V.E. Kagan, V. Castranova, A.A. Shvedova, Toxicology 2009, 257, 161-171.

[26] S. Tsuruoka, K. Takeuchi, K. Koyama, T. Noguchi, M. Endo, F. Tristan, M. Terrones, N. Saito, Y. Usui, D.W. Porter, V. Castranova, V. J. Phys.: Conf. Ser. 2013, 429, 01209.

[27]E.J. Pertersen, X. Tu, M.Dizdaroglu, M. Zheng, B.C.Nelson, Small 2013, 9, No.2, 205208.

[28]T. Peng, P. Zeng, D. Ke X. Liu X. Zhang, Energy Fuels 2011, 25, 2203-2210.

[29] Y. Takuma, Bulletin of TIRI 3, Tokyo Institute of Technology Ph.D. Dissertation $11719520102,2008$.

[30] K.R. Weeks, C.J. Bruell, N.R. Mohanty, Soil Sediment Contam. 2000, 9, 331-345.

[31] S. Tsuruoka, H. Matsumoto, K. Takeuchi, K. Koyama, N. Saito, Y. Usui, S. Kobayashi, E. Akiba, D. W. Porter, V. Castranova, F. Cassee, M. Endo, \#591 Society of Toxicology $53^{\text {rd }}$ Annual Meeting, Phoenix AZ, 2014.

[32] S. Tsuruoka, K. Takeuchi, K. Koyama, F. Tristan-Lopez, H. Matsumoto, N. Saito, Y. Usui, M. Endo, M. Terrones, D.W. Porter, V. Castranova, \#427, Society of Toxicology $52^{\text {nd }}$ Annual Meeting, San Antonio, TX, 2013.

[33]P.J. Krusic, E. Wasserman, P.N. Keizer, J.R. Morton, J.F. Preston, Science 1991, 254, $1183-1185$

[34]R. Saito, M.S. Dresselhaus, G. Dresselhaus, Physical Properties of Carbon Nanotubes, Imperial College Press, 1998.

[35]M. Ullah, A. pivrikas, N. Sedar Sariciftci, H. Sitter, "Chapter 8, Charge Transport in organic Diodes and OFETs: A Comparison”, H. Sitter eds., Small Organic Molecules on 
Surfaces, Springer Series in Materials Science 173, 2013.

[36]X. Shi, B. Jiang, J. Wang, Y. Yang, Carbon 2012, 50 1005-1013.

[37]P.J.F. Harris, Carbon Nanotube Science: Synthesis, properties and applications; Cambridge University Press, 2009, pp 146-178.

[38] S. Tsuruoka, Private memorandum to Prof. Natsume at Chiba University in Japan on EED and DOS at large chirality numbers, 2012.

[39]D.W. Porter, A.F. Hubbs, B.T. Chen, W. McKinney, R.R. Mercer, M.G. Wolfarth, L. Battelli, N. Wu, K. Sriram, S. Leonard, M. Andrew, P. Willard, S. Tsuruoka, M. Endo, T. Tsukada, F. Munekane, D.G. Frazer, V. Castranova, Nanotoxicology 2013, 7, 1179-1194.

[40] M.J. Martínez-Morlanes, P. Castell, P.J. Alonso, M.T. Martinez, J.A. Puértolas, Carbom 2012, 50, 2442-2452. 